polarization between $\overline{\mathrm{M}}$ and $\overline{\mathrm{X}}$, and $S_{7}$ is $S H$-like and is well above $S_{1}$ all along $\bar{\Gamma} \overline{\mathrm{X}}$.)

${ }^{17}$ G. P. Alldredge, R. E. Allen, and F. W. de Wette,
J. Acoust. Soc. Am. $\underline{49}, 1453$ (1971).

${ }^{18}$ R. E. Allen, G. P. Alldredge, and F. W. de Wette, Phys. Rev. B $\underline{4}, 1661$ (1971).

\title{
Surface Vibrations in a Model hcp Crystal*
}

\author{
R. E. Allen \\ Department of Physics, Texas A \& $M$ University, College Station, Texas 77843 \\ and \\ G. P. Alldredge and F. W. de Wette \\ Department of Physics, University of Texas, Austin, Texas 78712 \\ (Received 16 February 1972)
}

\begin{abstract}
The surface relaxation, vibrational modes, mean-square amplitudes of vibration, etc., have been calculated for the (0001) surface of a model hcp crystal in which the particles interact through a Lennard-Jones potential. The results are virtually indistinguishable from those for the (111) surface of a fcc crystal.
\end{abstract}

In a previous paper, ${ }^{1}$ we reported a study of the surface-mode spectrum for the (111) surface of a model fcc crystal in which the particles are taken to interact through a Lennard-Jones potential. Here we report a study of the surface vibrations in a model crystal with the same interaction potential which has an hcp structure and a (0001) surface. The two models differ only in that the former has an $A B C A B C .$. stacking and the latter has an $A B A B$... stacking.

We have followed the same procedure as described for the fcc crystals in our earlier papers $^{1,2}$ : First the static relaxation at the surface is determined by minimizing the static energy, then the vibrational frequencies and associated eigenvectors are determined, and finally physical quantities such as the mean-square amplitudes are calculated.

We find that the results of our calculations-for the relaxation, vibrational frequencies, meansquare amplitudes of vibration, etc., -are virtually indistinguishable from those obtained previously for the (111) surface of a fcc crystal. As one example, we show in Fig. 1 the results for the vibrational frequencies, which are almost exactly the same as those shown in Fig. 8 of Ref. 1. As another example, we mention that, contrary to an expectation that the short-wavelength surface modes might reflect the difference in stacking sequence
( $A B A B$ versus $A B C A B C$ ), the depth dependence of the surface modes $S_{1}, S_{2}, S_{3}$, and $S_{4}$ (when plotted for comparison with Fig. 9 of Ref. 1) displays no significant effect of the different stacking sequences.

On the basis of these results, we expect the same general behavior for surface vibrations at the (111) surfaces of monatomic fcc materials and (0001) surfaces of hcp materials.

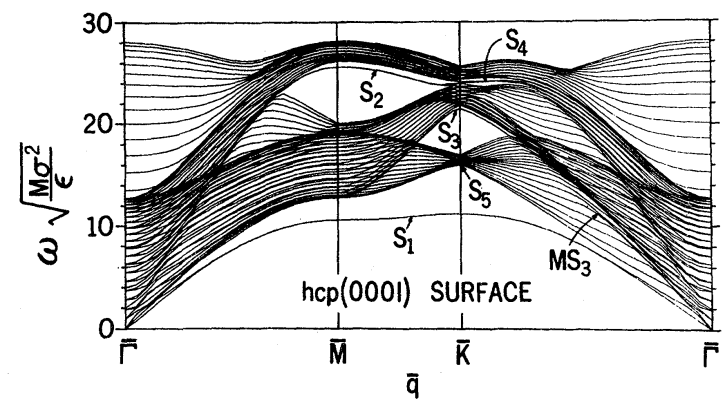

FIG. 1. Vibrational frequencies versus planar wave vector q for 21-layer slab with hcp structure and (0001) surfaces, determined in the same manner as in Ref. 1 with the surface relaxation taken into account. These results are virtually indistinguishable from those in Fig. 8 of Ref. 1 for a fcc structure and (111) surfaces. $(\bar{\Gamma}$ is the origin, and $\bar{\Gamma} \bar{M}$ and $\bar{\Gamma} \bar{K}$ are the [11 $\overline{2} 0]$ and [ $10 \overline{1} 0]$ directions, respectively.)

\footnotetext{
${ }^{*}$ Research sponsored by the U. S. Air Force Office of Scientific Research (AFSC) under Grant No. AFOSR 71-1973.

${ }^{1}$ R. E. Allen, G. P. Alldredge, and F. W. de Wette,
}

Phys. Rev. B $\underline{4}, 1661$ (1971).

${ }^{2}$ R. E. Allen and F. W. de Wette, Phys. Rev. 179,873 (1969). 